Canum 2000 : Actes du 32e Congrès national d'analyse numérique

A. Blouza, I. Danaila, P. Joly, S.M. Kaber, B. Lucquin, F. Murat \& M. Postel, Éditeurs

ESAIM: Proceedings, Vol. 11, 2002, 117-126

http://www.emath.fr/Maths/Proc/Vol.11/

(C)2002, Société de Mathématiques Appliquées et Industrielles, EDP Sciences

\title{
On the stability of viscous boundary layers
}

\author{
Emmanuel Grenier
}

Résumé. L'objet de l'exposé est de présenter quelques résultats d'instabilité d'écoulements de cisaillement pour les équations d'Euler et de Navier Stokes des fluides incompressibles.

Mots clés. Euler, Navier Stokes, Prandtl, stabilité

Abstract. The aim of this talk is to present some instability results for shear flows for Euler and Navier Stokes equations.

Key words. Euler, Navier Stokes, Prandtl, stability

AMS subject classification. 35J, 65N, 73C

\section{Introduction}

Let us consider the following Navier Stokes equations for incompressible fluids in a bounded two dimensionnal domain $\Omega \subset R^{2}$

$$
\partial_{t} u^{\nu}+\left(u^{\nu} . \nabla\right) u^{\nu}-\nu \Delta u^{\nu}+\nabla p^{\nu}=0,
$$

$$
\nabla \cdot u^{\nu}=0,
$$

$$
u^{\nu}=0 \quad \text { on } \quad \partial \Omega .
$$

The aim of this note is to describe the behavior of $u^{\nu}$ as $\nu \rightarrow 0$ in some particular cases. The main open problem is to know whether solutions $u^{\nu}$ of $(1,2,3)$ (for instance global regular solutions, see [9]) converge (and in what sense) to solutions of the Euler equations for incompressible ideal fluids

$$
\begin{gathered}
\partial_{t} u^{E}+\left(u^{E} \cdot \nabla\right) u^{E}+\nabla p^{E}=0, \\
\nabla \cdot u^{E}=0, \\
u^{E} \cdot n=0 \quad \text { on } \quad \partial \Omega,
\end{gathered}
$$

where $n$ is the outer normal of $\Omega$. The main problem in this singular limit is the change of the boundary condition (3) $\rightarrow(6)$, and we expect a boundary layer type behavior near $\partial \Omega$. Note that if $\Omega=R^{2}$ then, up to regularity assumptions on $u^{E}$, it is possible to prove that $u^{\nu} \rightarrow u^{E}$, for instance in $L^{\infty}\left(\left[0,+\infty\left[, L^{2}\left(R^{2}\right)\right)\right.\right.$ (see [8] for instance). Note also that (3) is the "worst" Article published by Eløp Sciences and available at http://www.edpsciences.org/proc or http:///dx.doi.org/10.1051/proc:2002033 
condition: convergence holds if we replace (3) by curl $u^{\nu}=0$ or by Navier conditions [3]. In [2], R.E. Caflisch and Sammartino proved convergence in small time for analytic initial data. As we will see, analyticity completly kills the underlying physical instabilities, therefore it is necessary to try to dismiss the analyticity assumption, and to work in Sobolev type spaces.

We will focus our analysis on $\Omega=T \times R_{+}$(periodic in the $x$ variable) or $R \times R_{+}$and on particular solutions of Navier Stokes equations, namely we will study the inviscid limit for shear layer profiles, that is for initial data of the form

$$
u_{0}^{\nu}(x, y)=\left(u_{s}(y / \eta), 0\right)
$$

where $u_{s}$ is some smooth function which satisfies $u_{s}(0)=0$ and which has a limit $u_{\infty}$ as $y \rightarrow+\infty$, and where $\eta \rightarrow 0$ as $\nu \rightarrow 0$ (in fact we will take $\eta=\sqrt{\nu}$ ). The formal analysis is straightforward. For such initial data, the solution of Navier Stokes equation $u^{\nu}(t, x, y)$ is of the form $\left(u_{s}(t, y / \eta), 0\right)$ where $u_{s}$ satisfies the heat equation

$$
\partial_{t} u_{s}-\frac{\nu}{\eta^{2}} \partial_{y y} u_{s}=0
$$

with boundary condition $u_{s}(t, 0)=0$. Note that the most interesting case arises for $\eta=\sqrt{\nu}$. Of course $u^{\nu} \rightarrow u^{E}$ where $u^{E}(t, x, y)=\left(u_{\infty}, 0\right)$ in any reasonable sense (in any norm which does not see the change (3) $\rightarrow(6))$. The main problem is to know whether this situation is stable or not, that is to know whether small perturbations of the initial data (7) will remain small and will lead to convergence to solutions of Euler equations close to $u^{E}$. This is true for analytic perturbations [2], but the situation is more intricated for Sobolev perturbations. Namely we will prove instability results in $H^{s}$ for $s>2$ for genuine Navier Stokes equations, nearly instability in $L^{\infty}$ up to an arbitrarly small forcing in Navier Stokes equations and will discuss the open problem of $L^{2}$ instability.

The first section is devoted to instability results for inviscid shear layers, the second section to $H^{s}$ instability results for Navier Stokes equations, and the last section to discussion on $L^{2}$ issues.

\section{Inviscid instability}

It could seem strange to begin the study by throwing away the viscous term, which a priori has a stabilizing role. However, as we will see in the next section, viscosity does not improve things, on the contrary it has a paradoxal destabilizing role !

In this section let $u_{s} \in C^{\infty}\left(R_{+}\right)$be a given function, and let $U_{s}(t, x, y)=\left(u_{s}(y), 0\right)$ be the corresponding shear layer profile. Note that $U_{s}$ is a stationnary solution of Euler equations. Linearized Euler equations around $U_{s}$ are

$$
\partial_{t} v+\left(U_{s} \cdot \nabla\right) v+(v \cdot \nabla) U_{s}+\nabla q=0,
$$

$$
\begin{gathered}
\nabla . v=0, \\
v . n=0 \quad \text { on } \quad \partial \Omega,
\end{gathered}
$$


Linear stability is a very old issue and Lord Rayleigh proved [10] that if $U_{s}$ is an unstable solution of Euler equation in the spectral sense (namely there exists an eigenvector $v$ and an eigenvalue $\lambda$ such that $v \exp (\lambda t)$ is solution of $(8,9,10)$ with $\operatorname{Re} \lambda>0)$ then it has an inflexion point (there is a point $y \geq 0$ such that $U_{s}^{\prime \prime}(y)=0$ ) and gave examples of linearly unstable profiles. This condition is not sufficient for instability and has been later refined by Fjortoft. Then spectral instability implies nonlinear instability

Theorem 2.1 [5] in $H^{2}$, [6] in $L^{\infty}$.

Let $\Omega=T \times R_{+}$or $R \times R_{+}$. Let $U_{s}$ be a smooth shear layer profile, unstable in the spectral sense. Then it is also nonlinearly unstable in the following sense: for every s arbitrarly large, there exists $C_{0}>0$, a family of solutions $u^{\eta}$ of Euler equations and a family of times $T^{\eta}$ such that

$$
\begin{gathered}
\left\|u^{\eta}(0, . . .)-U_{s}\right\|_{H^{s}} \rightarrow 0, \\
\left\|u^{\eta}\left(T^{\eta}, . . .\right)-U_{s}\right\|_{L^{\infty}} \geq C_{0}, \\
\left\|u^{\eta}\left(T^{\eta}, . . .\right)-U_{s}\right\|_{L^{2}} \geq C_{0},
\end{gathered}
$$

as $\eta \rightarrow 0$.

For instance $u_{s}(y)=\sin (y) \exp (-y)$ leads to a linearly and nonlinearly unstable shear layer profile, whereas $u_{s}(y)=1-\exp (-y)$ leads to a linearly and nonlinearly stable profile (use [1] for nonlinear instability).

\section{Viscous case}

\subsection{Linear instability}

The situation is more subtle for Navier Stokes equations, since against any expectation, the viscous term can have a destabilizing effect. For instance $u_{s}(y)=1-\exp (-y)$ is stable for Euler equations, but unstable for Navier Stokes equations for sufficiently small viscosity. This analysis has been fulfilled at the beginning of the century by Tollmien [4], who proved that any shear layer profile is linearly unstable for sufficiently small viscosity. More precisely let us consider the following linearized Navier Stokes equations around $U_{s}$

$$
\begin{gathered}
\partial_{t} v+\left(U_{s} . \nabla\right) v+(v \cdot \nabla) U_{s}-\nu \Delta v+\nabla q=0, \\
\nabla \cdot v=0, \\
v=0 \quad \text { on } \quad \partial \Omega .
\end{gathered}
$$

Let

$$
R e=\nu^{-1}
$$

be the Reynolds number. If we introduce a stream function for $v$ and take its Fourier transform in the $x$ variable, we are lead to look for solutions of the form

$$
v(t, x, y)=\exp (i k x)\left(\begin{array}{c}
\Psi^{\prime}(t, y) \\
-i k \Psi(t, y)
\end{array}\right)
$$


The spectrum of $(11,12,13)$ is thus obtain by looking at solutions of the form

$$
v(t, x, y)=\exp (i k(x-c t))\left(\begin{array}{c}
\Psi^{\prime}(y) \\
-i k \Psi(y)
\end{array}\right) .
$$

which leads to the classical Orr-Sommerfeld equations

$$
-(i k R e)^{-1}\left(\partial_{y}^{2}-k^{2}\right)^{2} \Psi+\left(u_{s}-c\right)\left(\partial_{y}^{2}-k^{2}\right) \Psi=\Psi v_{s}^{\prime \prime}
$$

with boundary conditions $\Psi(0)=\Psi^{\prime}(0)=\lim _{+\infty} \Psi=0$. As $\nu \rightarrow 0$, Orr Sommerfeld equation can be studied as a viscous perturbation of Rayleigh equation

$$
\left(u_{s}-c\right)\left(\partial_{y}^{2}-k^{2}\right) \Psi=\Psi v_{s}^{\prime \prime}
$$

with boundary conditions $\Psi(0)=\lim _{+\infty} \Psi=0$. Rayleigh equation can also be obtained by looking for solutions of linearized Euler equations (8) of the form (14). Two cases arise depending on whether $\left(u_{s}(y), 0\right)$ is spectrally stable for Euler equations or not.

\subsubsection{Unstable inviscid profiles}

If $\left(u_{s}(y), 0\right)$ is spectrally unstable for Euler equations, there exists $c, k$ and $\Psi$ solutions of (16) with $\operatorname{Im} c>0$. As $\Psi^{\prime}(0)$ has no reason to be zero, a boundary layer appears near $y=0$ in the solutions $\Psi^{\nu}$ of Orr Sommerfeld equation (15). As $v_{s}(0)=0$, the equation of this layer is

$$
(i k R e)^{-1} \partial_{y}^{4} \Psi^{b}=-c \partial_{y}^{2} \Psi^{b}
$$

and its size is of order $R e^{-1 / 2}$. A simple perturbative analysis then shows that for $R e$ large enough there exists a solution $c_{\nu}, k$ and $\Psi_{\nu}$ of (15) with $c_{\nu} \rightarrow c$ and with

$$
\Psi^{\nu} \sim \sum_{j \geq 0} \nu^{j} \Psi^{i n t, j}(y)+\sum_{j \geq 0} \nu^{j} \Psi^{b, j}\left(R e^{1 / 2} y\right)
$$

This implies in particular that $\left(v_{s}(y), 0\right)$ is linearly unstable at sufficiently large Reynolds numbers. Note the boundary layer behavior of $\Psi^{\nu}$. Moreover the growth rate of the most unstable mode converges to the growth rate of the most unstable mode of Rayleigh equation (which is positive) as $R e$ goes to infinity.

\subsubsection{Stable inviscid profiles}

The case where $\left(v_{s}(y), 0\right)$ is spectrally stable for Euler equations is more subtle since we have to perturbe the essential spectrum of (16), which consists of a part of the real axis. Namely Orr Sommerfeld equation is a very singular perturbation of Rayleigh equation if $c \in R$ and lies in the range of $u_{s}$. Let $c \in R$ and let $y_{c}$ such that $u_{s}\left(y_{c}\right)=c$. Then at $y_{c},(16)$ has a singular point. By classical Fuchs theory there exists a smooth solution $\Psi_{1}$ of (16) which vanishes at $y_{c}$, and a singular solution $\Psi_{2}$ which has a logarithmic branch at $y_{c}$. When we add the viscosity and try to construct a solution of (15) starting from a solution of (16) without taking care of the boundary 
conditions, we are in big troubles for $\Psi_{2}$ (it is straightforward for $\Psi_{1}$ ). Around $y_{c}$ appears a layer (called critical layer) of size $(k R e)^{-1 / 3}$, of equation of the form

$$
\left(\partial_{Y}^{2}-Y\right) \partial_{Y}^{2} \Psi=0
$$

where $Y=(k R e)^{-1 / 3}\left(y-y_{c}\right)$ and its solutions involve Airy's functions, which leads to very heavy computations. Moreover, as previously, the boundary conditions change from Orr Sommerfeld to Rayleigh, therefore a boundary layer appears near $y=0$, of size of order $(k R e)^{-1 / 2}$. In some cases this layer and the critical layer are not separated.

The result is that provided $u_{s}^{\prime}(0) \neq 0$ and $u_{s}^{\prime \prime}(0) \neq 0$ (else the situation is technically even worse), there exists $\nu_{c}>0$ such that $(11,12,13)$ is linearly stable if $\nu>\nu_{c}$ and linearly unstable if $\nu<\nu_{c}$. Moreover the growth rate of the most unstable mode behaves like $R e^{-1 / 2}$ as $R e$ goes to infinity. This leads to

Any shear flow is linearly unstable at sufficiently large Reynolds number.

\subsection{Nonlinear instability in $H^{s}$ norm of shear layers}

Starting from this linear instability we can show that shear layer profiles are very sensitive to arbitrarly small and smooth perturbations, which leads to instability results in strong norm. Let us start from a smooth shear layer profile $u_{s}$ such that $\left(u_{s}(y), 0\right)$ is a spectrally unstable profile for Euler equations, and let us study the behavior of $\left(u_{s}(y / \sqrt{\nu}), 0\right)$ for $(1,2,3)$. The first step is to rescale space and time in order to deal with a fixed profile. Let $\tilde{x}=x / \sqrt{\nu}, \tilde{y}=y / \sqrt{\nu}, \tilde{t}=t / \sqrt{\nu}$. In these new variables, Navier Stokes equations are unchanged, except the viscous term which turns to $\sqrt{\nu} \Delta$. We will forget the tildes from now on.

Then there exists a solution of $(8,9,10)$ of the form $v(t, x, y)=\exp (i k(x-c t)) \tilde{v}(y)$ (where $\tilde{v}$ is a smooth vector valued function) with $\operatorname{Im} c>0$, and a family of solutions of $(11,12,13)$ with viscosity $\nu^{1 / 2}$ of the form $v^{\nu}(t, x, y)=\exp \left(i k\left(x-c_{\nu} t\right)\right) \tilde{v}^{\nu}(y)$ where $c_{\nu} \rightarrow c$ and where $v^{\nu}$ is of the form

$$
v^{\nu} \sim \sum_{j \geq 0} \sqrt{\nu}^{j} v^{i n t, j}(y)+\sum_{j \geq 0} \sqrt{\nu}^{j} v^{b, j}\left(y / \nu^{1 / 4}\right)
$$

where $v^{i n t, 0}=v$ and where $v^{b, 0}$ is not identically zero (boundary layer). Let $N$ and $M$ be two large integers. Let $\delta=\nu^{M}$. Starting from $v^{\nu}$ we can construct a sequence of approximate solutions of $(11,12,13)$

$$
u^{a p p}(t, x, y)=\left(u_{s}(t, y), 0\right)+\delta \sum_{j=0}^{N} \sqrt{\nu}^{j} v^{i n t, j}(t, x, y)+\delta \sum_{j=0}^{N} \sqrt{\nu}^{j} v^{b, j}\left(t, x, y / \nu^{1 / 4}\right)+c . c .
$$

(c.c. stands for "complex conjugate", and $u^{i n t, j}(0, . .)=.u^{i n t, j}(.,$.$) , and similarly for u^{b, j}$ ) in the sense that $u^{a p p}$ is divergence free, satisfies the boundary conditions and

$$
\partial_{t} u^{a p p}+\left(u^{a p p} \cdot \nabla\right) u^{a p p}-\sqrt{\nu} \Delta u^{a p p}+\nabla p^{a p p}=\nu^{M} \nu^{N / 4-4} R^{\nu}
$$

where $\left\|R^{\nu}(t, ., .)\right\|_{L^{2}} \leq C e^{C_{0} t}$ for some constant $C_{0}$. Now let $u^{\nu}$ be the solution of Navier Stokes equations with viscosity $\sqrt{\nu}$ and initial data $u^{a p p}(0, .,$.$) . Let v^{\nu}=u^{\nu}-u^{a p p}$ which satisfies

$$
\partial_{t} v^{\nu}+\left(u^{\nu} . \nabla\right) v^{\nu}+\left(v^{\nu} . \nabla\right) u^{a p p}-\sqrt{\nu} \Delta v^{\nu}+\nabla q^{\nu}=-\nu^{M+N / 4-4} R^{\nu}
$$


together with divergence free and boundary conditions. A simple $L^{2}$ estimate gives

$$
\partial_{t} \int \frac{\left|v^{\nu}\right|^{2}}{2}+\sqrt{\nu} \int\left|\nabla v^{\nu}\right|^{2} \leq\left(1+\left\|\nabla u^{a p p}\right\|_{L^{\infty}}\right) \int \frac{\left|v^{\nu}\right|^{2}}{2}+\nu^{2 M+N / 2-2} \int\left|R^{\nu}\right|^{2},
$$

which, using (17) gives

$$
\partial_{t} \int \frac{\left|v^{\nu}\right|^{2}}{2} \leq C\left(1+\nu^{M} \nu^{-1 / 4} \exp (k \operatorname{Im} c t)\right) \int \frac{\left|v^{\nu}\right|^{2}}{2}+\nu^{2 M+N / 2-2} \int\left|R^{\nu}\right|^{2} .
$$

This energy estimate is usefull as long as $1+\nu^{M} \nu^{-1 / 4} \exp (k \operatorname{Im} c t)$ remains bounded, that is as long as

$$
t \leq T_{\nu}=\left(M-\frac{1}{4}\right) \frac{\log \nu^{-1}}{k \operatorname{Im} c} .
$$

After this time, it is useless and we completly loose the control on $u^{\nu}$. If we stop at $T_{\nu}$ then $u^{a p p}$ is of the form

$$
u^{a p p}=\left(u_{s}(y), 0\right)+\nu^{1 / 4} v^{i n t, 0}(y)+\nu^{1 / 4} v^{b, 0}\left(y / \nu^{1 / 4}\right)+\text { c.c. }+O\left(\nu^{1 / 2}\right) .
$$

This leads to the following result, going back to the initial coordinates

Theorem 3.1 Nonlinear instability in $H^{2}$ [7].

Let $\left(u_{s}(y), 0\right)$ be a smooth boundary layer profile, unstable for Euler equations. Let $u_{s}(t, y)$ be the solution of the heat equation with Dirichlet boundary condition. Then $\left(u_{s}(t, y / \sqrt{\nu}), 0\right)$ is a nonlinearly unstable solution of Navier Stokes equations with viscosity $\nu$ in the following sense: for every s and $N$ arbitrarly large, there exists a constant $C_{0}>0$, a sequence of times $T_{\nu}$ and of solutions $u^{\nu}$ such that

$$
\begin{gathered}
\left\|u^{\nu}(0, ., .)-\left(u_{s}(y / \sqrt{\nu}), 0\right)\right\|_{H^{s}} \leq \nu^{N} \\
\left\|u^{\nu}\left(T^{\nu}, . . .\right)-\left(u_{s}\left(T^{\nu}, y / \sqrt{\nu}\right), 0\right)\right\|_{H^{2}} \rightarrow+\infty \\
\left\|u^{\nu}\left(T^{\nu}, . . .\right)-\left(u_{s}\left(T^{\nu}, y / \sqrt{\nu}\right), 0\right)\right\|_{L^{2}} \geq C_{0} \nu^{1 / 2} \\
T^{\nu} \rightarrow 0
\end{gathered}
$$

as $\nu \rightarrow 0$.

This theorem has several consequences. First it gives examples of unstable solutions of Navier Stokes equations. Second it says that it is impossible to get an asymptotic expansion of the form

$$
u^{\nu}(t, x, y) \sim \sum_{j \geq 0} \sqrt{\nu}^{j} u^{i n t, j}(t, x, y)+\sum_{j \geq 0} \sqrt{\nu}^{j} u^{b, j}(t, x, y / \sqrt{\nu})
$$

for solutions $u^{\nu}$ of $(1,2,3)$ as $\nu \rightarrow 0$, in $H^{s}$ for $s \geq 2$. It even says that we can not expect that

$$
u^{\nu}(t, x, y)=u^{\text {Euler }}(t, x, y)+u^{B L}(t, x, y / \sqrt{\nu})+o\left(\nu^{1 / 4}\right)_{L^{\infty}}
$$

where $u^{E u l e r}$ is solution of Euler equations and $u^{B L}$ describes a boundary layer type behavior of $u^{\nu}$. Note that there is also no hope to get asymptotic expansions of that type even if we replace $\sqrt{\nu}$ 
by another boundary layer scale. This theorem also describes precisely what happens: a sublayer of size $\nu^{3 / 4}$ appears near the boundary, inside the classical $\nu^{1 / 2}$ layer.

Note that in the initial $t, x, y$ variables, the instable perturbations have a very high spatial frequency, of order $1 / \sqrt{\nu}$. Therefore, provided we multiply them by some power of $\nu$ they can be made as small as we want in Sobolev spaces at $t=0$. However in analytic type spaces, this not sufficient to keep them bounded, and we have to multiply them by some $\exp (-1 / \sqrt{\nu})$ factor. In this case instability appears after a time of order $O(1)$ since they have an exponential growth. This leaves the place for a convergence result in analytic spaces, which was done in [2].

\subsection{Nonlinear instability result in $L^{\infty}$}

As we loose control on the $L^{2}$ norm of $u^{\nu}-u^{a p p}$ because of very large gradients in the sublayer, it is very difficult to obtain instability results in lower norms. To go further we will allow a small force term in Navier Stokes equations. More precisely we can construct a very accurate approximate solution, but this solution is unstable, therefore it is useless to try to get energy estimates. To use this approximate solution we simply define the force $f^{\nu}$ as being the remainder term $R^{\nu}$. Now the approximate solution is a true solution of Navier Stokes equation with source term $R^{\nu}$ ! This leads to

Theorem 3.2 "Almost" nonlinear instability in $L^{\infty}$ with forcing.

Let $\left(u_{s}(y), 0\right)$ be a smooth boundary layer profile, unstable for Euler equations. Let $u_{s}(t, y)$ be the solution of the heat equation with Dirichlet boundary condition. Then $\left(u_{s}(t, y / \sqrt{\nu}), 0\right)$ is a nonlinearly unstable solution of Navier Stokes equations with viscosity $\nu$ in the following sense: for every $s$ and $N$ arbitrarly large and for $\alpha>0$ arbitrarly small, there exists a sequence of times $T_{\nu}$, a sequence of forces $f^{\nu}$ and a sequence of solutions $u^{\nu}$ of Navier Stokes equations with forcing term $f^{\nu}$

$$
\partial_{t} u^{\nu}+\left(u^{\nu} . \nabla\right) u^{\nu}-\nu \Delta u^{\nu}+\nabla p^{\nu}=f^{\nu},
$$

such that

$$
\begin{gathered}
\left\|u^{\nu}(0, . . .)-\left(u_{s}(y / \sqrt{\nu}), 0\right)\right\|_{H^{s}} \leq \nu^{N} \\
\left\|f^{\nu}\right\|_{L^{\infty}\left(\left[0, T^{\nu}\right], H^{s}\right)} \leq \nu^{N} \\
\left\|u^{\nu}\left(T^{\nu}, ., .\right)-\left(u_{s}\left(T^{\nu}, y / \sqrt{\nu}\right), 0\right)\right\|_{L^{\infty}} \geq \nu^{\alpha} \\
T^{\nu} \rightarrow 0
\end{gathered}
$$

as $\nu \rightarrow 0$.

This theorem almost gives an instability result in $L^{\infty}$, but the trick fails in proving genuine $L^{\infty}$ instability.

\section{Open problems: $L^{2}$ issues}

\subsection{A result of $\mathrm{T}$. Kato}

In a wonderfull paper, T. Kato [8] proved 
Theorem 4.1 T. Kato [8].

Let $u$ be a smooth solution of Euler equation in a bounded domain $\Omega$. Let $u^{\nu}$ be a sequence of solutions of Navier Stokes equations, such that

$$
\left\|u^{\nu}(0, . . .)-u(0, ., .)\right\|_{L^{2}} \rightarrow 0 .
$$

Then $u^{\nu} \rightarrow u$ in $L^{\infty}\left([0, T], L^{2}(\Omega)\right)$ if and only if for every $C>0$,

$$
\nu \int_{0}^{T} \int_{d(x, \partial \Omega)<C \nu}\left\|\nabla u^{\nu}\right\|^{2} d x d t \rightarrow 0
$$

as $\nu \rightarrow 0$.

The principle of the proof is to construct an approximate solution $u^{a p p}$ of Navier Stokes equations starting from $u$ and adding a boundary layer of size $\nu$, without taking care of its equation, since the boundary layer has a negligible $L^{2}$ energy. Note that the integral over the dissipation holds over a strip $d(x, \partial \Omega)<C \nu$ and not $C \sqrt{\nu}$ : T. Kato adds boundary layers of size $\nu$ and not of the classical size $\sqrt{\nu}$.

A classical point of view is that as the boundary layer becomes instable, it splits from the boundary, enters into the domain and probably disturbs it completly. Kato's result leads to a change of point of view: if we want to prove that solutions of Navier Stokes equations do not converge to solutions of Euler equations in $L^{\infty}\left(L^{2}\right)$, instead of trying to prove that the boundary layer splits from the boundary (which is almost hopeless since in any case the energy of the boundary layer is negligible), we have to try to prove that something bad happens at a distance $\nu$ of the boundary. Hence instead of looking at distances large with respect to $\sqrt{\nu}$ we have to look at smaller and smaller distances, nearer and nearer the boundary

\subsection{Toward a boundary layer cascade?}

As described in the previous sections if we start with an initial layer of size $\sqrt{\nu}$, a sublayer of size $\nu^{3 / 4}$ appears in times of order $T_{1}=\sqrt{\nu}$. The analysis of section 3.2 then stops when large gradients appear in this sublayer. With a small force in section 3.3 we were able to go a little further in time. If we look carefully at what happens then, we see a disturbed flow of size $\sqrt{\nu}$ (unstable initial boundary layer) and a sublayer of typical length $\nu^{3 / 4}$ and of amplitude almost $O(1)$. The gradients in this layer are then of order almost $O\left(\nu^{-3 / 4}\right)$. Let us introduce the Reynolds number $R e_{2}$ of this sublayer (product of its size by the typical velocity, divided by the viscosity). It equals

$$
\operatorname{Re}_{2}=\frac{\nu^{3 / 4} \times 1}{\nu}=\nu^{-1 / 4}
$$

and therefore goes to $+\infty$ as $\nu$ goes to 0 . Hence provided $\nu$ is small enough, the sublayer is itself linearly unstable. An arbitrarly small well choosen force can then destabilize it, within times much smaller than the time scale $T_{1}$, and we can reproduce the analysis of the previous sections. This again creates a subsublayer of size $R e_{2}^{-1 / 2} \nu^{3 / 4}=\nu^{7 / 8}$. This construction is linked to the so called "triple deck". 
Reproducing theorem 3.2 we can iterated the construction. A sequence of boundary layers of sizes

$$
\nu^{\frac{2^{N}-1}{2^{N}}}
$$

appear successively, a sequence of sizes with precisely tends to $\nu$ in link with T. Kato's theorem. More precisely the construction can be iterated as soon as the $N^{\text {th }}$ Reynolds number is large enough, that is until $\nu^{\left(2^{N}-1\right) / 2^{N}}=C \nu$, where $C$ is of order 100 . For a given $\nu$ there are only a finite numbers of boundary layers, of order

$$
\log \left(\frac{\log R e}{\log C}\right) / \log 2
$$

Therefore the triple deck appears at Reynolds of order $10^{12}$, the fourth layer at $R e \sim 10^{24}$ and the fifth at $R e \sim 10^{48}$ which seems rather unphysical Reynolds numbers.

At the end of this formal construction, we get a sequence of approximate solutions and a time $T^{\nu}$ such that at $T^{\nu}$

$$
\int_{d(x, \partial \Omega) \leq C \nu \log \nu^{-1}}\left\|\nabla u^{a p p}\right\|^{2} d x \leq \frac{C}{\log \nu} .
$$

Note the $\log$ factor which differs from Kato's criterium (in addition to the time integral of course). This indicates that if convergence holds true, it is not a very fast one, only as $(\log \nu)^{-1}$.

\subsection{Physical meaning}

The previous construction is rather unphysical, since to get three sublayers we have to go to Reynolds numbers of order $10^{12}$ and even more, which is physically not realistic. This in particular means that interesting mathematical behaviour appear continuously as the Reynolds increases to infinity. To handle this mathematical complexity is necessary if we want to get convergence, or non convergence in $L^{2}$. However, it is far from the behavior of real fluids ! To sum up, the physics lies in large but not huge Reynolds number.

In fact theorem 3.2 describes the transition between the laminar Prandtl boundary layer and turbulent phenomena, a transition which occurs for Reynolds of order $10^{6}$ or $10^{7}$ and which has been observed for a long time. Note also that Kato criterium is linked to the viscous sublayer considered in the theory of turbulent boundary layers (log laws for instance).

\section{References}

[1] V.I. ARNOLD. Sur la géométrie différentielle des groupes de Lie de dimension infinie et ses applications à l'hydrodynamique des fluides parfaits. Annales de l'Institut Fourier, 16, 1969, 319-361.

[2] M. Sanmmartino, R. Caflisch. Zero viscosity limit for analytic solutions of the Navier Stokes equation on a half space. I et II. Comm. Math. Phys., 192, 1998, 433-461 et 463-491.

[3] T. Clopeau, A. Mikelić, R. Robert. On the vanishing viscosity limit for the $2 \mathrm{D}$ incompressible Navier-Stokes equations with the friction type boundary conditions. Nonlinearity , 11, 1998, 1625163. 
[4] P.G. DRAZIN, W.H. ReID. Hydrodynamic stability. Cambridge monographs on mechanics and applied mathematics, Cambridge University Press, 1981.

[5] S. Friedlander, W. Strauss, M. Vishik. Nonlinear instability in an ideal fluid. Ann. Inst. H. Poincaré Anal. Non Linéaire, 14, 1997, 187-209

[6] E. GreniER. On the stability of boundary layers of Euler equations. to appear in Journal Diff. Equ. .

[7] E. GREnIER. On the nonlinear instability of Prandtl equations. to appear in Comm. Pure and Applied Math.

[8] T. Kato. Remarks on zero viscosity limit for nonstationary Navier Stokes flows with boundary. Seminar on nonlinear partial differential equations (Berkeley, Calif., 1983), Springer, Math. Sci. Res. Inst. Publ., 2, 1984, 85-98.

[9] J. Leray. Sur le mouvement d'un fluide visqueux emplissant l'espace. Acta Math., 35, 1936, 139149.

[10] Lord Rayleigh. On the instability or instability of certain fluid motions. Proc. London Math. Soc., 11, 1880, 57-79.

Emmanuel Grenier

UMPA

Ecole Normale Supérieure de Lyon

46 allée d'Italie

69364 Lyon Cedex 07, France

mailto:egrenier@umpa.ens-lyon.fr 